\title{
Interference effect during word-task and colour-task in incongruent stroop-task
}

\author{
Christianus Frederick Hotama, Hanung Adi Nugroho*, Indah Soesanti, Widhia K.Z Oktoeberza \\ Department of Electrical Engineering and Information Technology, Faculty of Engineering, Universitas Gadjah Mada \\ Jalan Grafika 2, Kampus UGM, Yogyakarta 55281, Indonesia
}

Article history:

Received: 14 October 2017 / Received in revised form: 13 November 2017 / Accepted: 19 November 2017

\begin{abstract}
Stroop-task is one of the most popular studies to check the ability of decision-making and cognitive process during high interference activity in the brain. In the incongruent Stroop-task, the difference between the colour that we read and the colour that we see produces high interference activities in the brain. This research aims to analyse the activity differences in each part of the brain during colour-task and word-task. This study investigates how well the ability of decision-making and cognitive process during high interference activities that occur in the brain. Electroencephalography (EEG) can record brain activities by recording the brain waves. The results show that recognising the colour is more difficult than that of the written words in the Stroop-task as indicated by statistical test with $t$-value greater than threshold value ( $\mathrm{t}>2.0027)$ and significant level of 0.05 . This study concludes that the colour-task gives more interference effect than the word-task. The more interference effect is produced, the more wrong decision-making is obtained.
\end{abstract}

Keywords: Brain wave; cognitive process; decision-making; Stroop-task.

\section{Introduction}

Stroop-task is a type of test that makes interference in the brain because the colour and the written word are not always the same. Commonly, in a normal study, it is more difficult to recognise the colour that we see (colour-task) than to recognise the colour that we read (word-task). There are three types of Stroop-task, i.e. neutral, congruent and non-congruent Stroop-tasks [1]. The purpose of the Stroop-task is to evaluate the ability of decision making and cognitive process during interference activities inside the brain [2].

There are two parts of the brain, namely anterior cingulate cortex and dorsolateral prefrontal cortex that are strongly involved in the Stroop-task. Anterior cingulate cortex (ACC) is the part to do error detection. It is located in the Brodmann areas no. 24, 32 and 33 as depicted in Fig. 1. The main function of ACC is to control the anatomy process. In Strooptask, this part is useful for error detection and conflict detection with human decisions. Dorsolateral prefrontal cortex (DLPFC) is the part of the primate cortex which can be found in both human and primate animal's brains. This part affects the ability of human working memory. This part is active when someone is conducting an activity, cognitive flexibility and another complex mental activity [3-6]. Both ACC and DLPFC involve in brain cognitive process [6]. The cognitive control during Stroop-task has significant effect on

* Corresponding author. Tel.: +62-274-552305; fax: +62-274-552305

Email: adinugroho@ugm.ac.id. the activity of DLPFC [7]. In the Stroop-task, DLPFC helps ACC to show a lot of activities during congruent and incongruent Stroop-task [8].

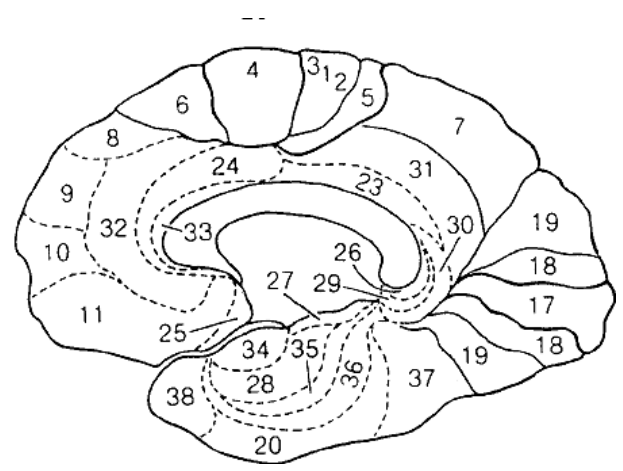

Fig. 1. Brodmann's areas of the cortex [9]

Non-invasive methods have advantages to measure the brain activities without making any scars in the human body. Electroencephalography (EEG) is one of the non-invasive devices to measure brain activities. This device uses brainwave signals as a reference to measure brain activities. To get the brain-wave signals, the EEG measures brain's spontaneous electrical activities in a period time [10].

Event related potential (ERP) is a method to evaluate the brain function. In some studies, ERP technique is one of the common methods to measure the brain response during cognitive task [11-13]. EEG data is useful in brain computer 
interface $(\mathrm{BCI})$ to make direct communication pathway between brain and external devices [1, 14-16].

Schack et al. [17] analysed EEG signals by comparing the brain activities during congruent to that of non-congruent in normal subject. The mean-coherent analysis in this experiment showed that there was significant activity difference between congruent and non-congruent Stroop-tasks around frontal lobe region. This study also showed that 13-20 $\mathrm{Hz}$ brain wave frequencies were strongly active during incongruent task. However, this task used verbal response for each case. As predicted, the tongue movement affected EEG recording data.

Another experiment by Peterson et al. [3] used fMRI to find the difference in brain activities during normal, congruent and non-congruent Stroop-tasks. Since fMRI can produce good image results of brain activities, they did some contrast comparison between each task in each brain region. This study found that incongruent Stroop-task produced reliable interference effect. Moreover, the interference during incongruent Stroop-task had some effects on the accuracy of the decision-making.

The verbal response was changed to movement response in study by Szúcs et al. [18] This study aimed to reduce the effect of tongue muscle movement for the EEG data. This study showed there was more activity in motor region during incongruent Stroop-task than that of congruent Stroop-task. However, previous studies only compared the brain activities among tasks that do not give much interference and that of gave a lot of interferences in the brain. Therefore, these studies only compared the decision-making process in two different conditions.

With some modifications from previous studies, this research compares the word-task and the colour-task. Both of these tasks are parts of the non-congruent Stroop-task. Therefore, both the tasks contain high interference level. This study investigates the condition that gives more interference effect leading to confuse the subjects more during decisionmaking process without considering the relation between times to finish the task with the frequency. In this study, the subject response is changed from verbal response to handmovement response in order to reduce the effect of non-reflex muscle movement. Since previous studies showed that the ageing effect was related with brain-wave activity, visual attention and working memory [19-21], the subjects are limited to people with the same range of age.

Brain-wave frequency occurs when the brain is doing some activities. In normal subjects, the range of the frequency is from 0.5 to $50 \mathrm{~Hz}$. There are five common types of brainwave frequency. Each frequency occurs in any human activity. Only some subjects who have overrated ability can produce a brain wave with more than $50 \mathrm{~Hz}$ (hyper-gamma range $[22,23]$. Beta-wave frequency range is also known as concentration wave. This type of brain-wave occurs when someone is doing an activity that needs high concentration, such as analysis process, managing information and finding a solution. This type of brain-wave can affect someone productivity [24]. The structure of this study is organised as follows. Section II describes the materials and experimental method. Section III presents the results and discussion followed by conclusion in Section IV.

\section{Materials and Methods}

\subsection{Subjects}

In this research, data were taken from seven normal subjects, all males and right-handed, with the range of age from 21 to 24 from Neuroimaging and Neuroinformatics laboratory, Kyushu University. For every task, the incongruent Stroop-task by Zysett et al. [25] was used. EEG POLYMATE $\mathrm{V}$, an EEG portable was used to record the brain-wave signal. The type of probe for each channel was active electrode. The 10-20 EEG standards was used as the reference for EEG measurement. The resistance level for the entire channels must below 10,000 ohm. There were three points used as references in this study. These points were located at the nasion part, left and right ears.

\subsection{Task Design}

In the interface of the tasks as shown in Fig. 2, the subject saw both WORD1 (upper word) and WORD2 (lower word). The tasks were designed with Visual Studio 2010 with BASIC language programming. The subject needed to make a decision if there was equal or non-equal case by pressing the mouse to the decision button.

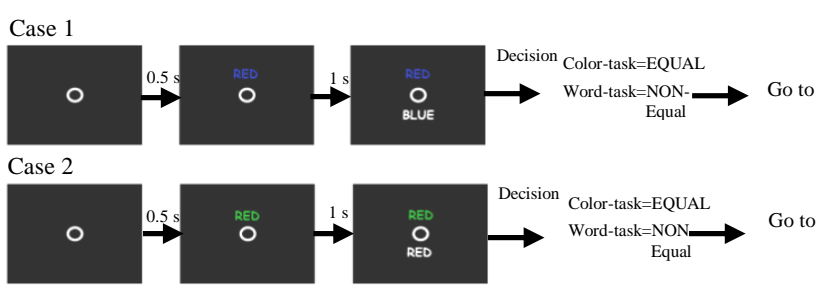

Fig. 2. The scheme of the Stroop-task recognition and decision making

After the subject was ready, the monitor showed black screen with little dot in the middle of the screen. This little dot was used as the attention point to the subject so that he or she could not easily lost his or her focus. After 0.5 second, WORD 1 appeared. The colour of WORD1 appeared randomly, and so did the written word. However, only 4 primary colours were used in this test. They were red, green, yellow and blue. WORD 1 was the Stroop-word, in which the seen colour was not always same as the written colour. After 1 second, WORD2 was shown in the monitor. WORD2 was a neutral word that always had white colour, but the written word appeared randomly. After WORD2 appeared, the subject took his or her time to make a decision.

There were two types of task conducted by the subject during the experiment, i.e. the word-task and the colour-task. For the word-task, the subject needed to compare the word that they read in WORD1 with the word that they read in WORD2. If the word that they read in WORD1 was the same as that of in WORD2, the subject needed to press EQUAL button. On the other hand, if it was not the same, the subject needed to press NON-EQUAL button.

For the colour-task, the subjects needed to compare the colour that they saw in WORD1 with that of in WORD2. If the colour that they see in WORD1 was the same as that of WORD2, the subject needed to press EQUAL button. Otherwise, the subject needed to press NON-EQUAL button. 
In Fig. 2, if the case 1 was part of the colour-task, the subject needed to answer equal, because the colour of WORD1 was the same as the written word in WORD2 (blue and blue). If case 1 was part of the word-task, the subject needed to answer non-equal, because the written word in WORD1 was not the same as the written word in WORD2 (red and blue). On the other hand, if the case 2 was part of the colour-task, the subject needed to answer non-equal, because the colour of WORD1 was the same as the written word in WORD2 (green and red). If case 2 was part of the word-task, the subject needed to answer equal, because the written word in WORD1 was not the same as the written word in WORD2 (red and red).

After the subject made the decision, the next case appeared with the same procedure with the case before. After the subject finished his or her 25th case of the Stroop-task, the subject was given a 15 seconds break. The monitor showed a countdown time until the next set of the task. With the same pattern, the subject underwent the same procedure to make a decision.

The time allowance for each task for each subject is shown in Fig. 3. The subject has gotten 4 trials for each task. Each trial contained 25 cases of Stroop-task. The time to make a decision was unlimited, but all of the trials showed that the subject needed from 1 to 2 minutes for each trial. There were 15 seconds break between each trial. This break was useful to decrease the sequence mistake and made the subject more relaxed for the next trial.

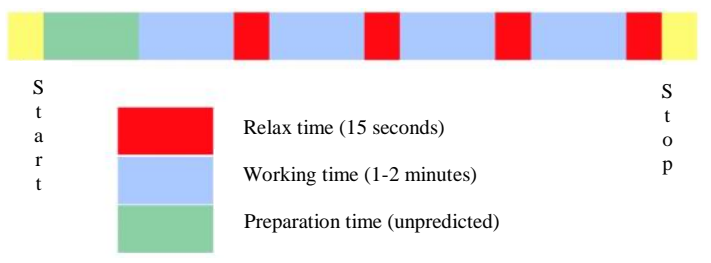

Fig. 3. Time allowance for 1 task

Band pass filter was used to reduce the wave that has the frequency outside of the brain wave range. Notch filter was used to reduce the probability signal of electricity source mixed with the brain wave signal. The noise occurring from the muscle movement, such as eye blink or eye movement was reduced by using independent component analysis (ICA) decomposition.

\subsection{Data EPOCHs}

To accurately get ERP results, the length time of the data needs to be same. Therefore, it needs to adjust the length of each data. This study extracted 2 EPOCHs from each trial. Fig. 4 shows how to extract the EPOCHs from a set of each task. Baseline data was used as the reference data when subject was still in the concentration mode without a lot of movements. Resting time between each trial was the best duration time in this experiment to take role as baseline time. The recorded EEG data showed the steadiest graph between another range times.

Each EPOCH has 20 seconds of EEG data. The EPOCH data were taken when the subject conducted the word-task or the colour-task. The range of the time was taken from 20 seconds before resting time for the first scheme, and 40 seconds before resting time until 20 seconds before resting time for the second scheme. Hence, each trial produced 2 EPOCHs.

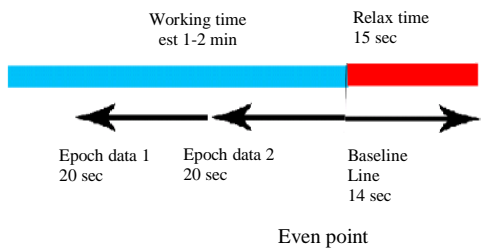

Fig. 4. EPOCH scheme for data analysis

This study used EEGLAB toolbox v 12.0.5 to analyse and to plot the ERP results and data signal processing. This toolbox was built by SCCN, University of San Diego, CA [26].

\section{Results and Discussion}

\subsection{Behavioural Result}

From the seven subjects, 700 cases of word-task and colour-task were solved. The percentage of right number of right answer for word-task was $99.2 \%(\mathrm{SD}=0.79)$ and colour task was $79.14 \%(\mathrm{SD}=2.91)$. The average time for a subject to finish one set of the task (25 cases of either word-task or colour-task) during colour task was 106.79 seconds (4.27 seconds for one case) and word task is 68.04 seconds $(2.72$ seconds for 1 case).

\subsection{EEG Result}

Fig. 5 shows the frequency spectrum of brain wave that has been recorded during colour-task (green) and word-task (blue) in decibel $(\mathrm{dB})$.

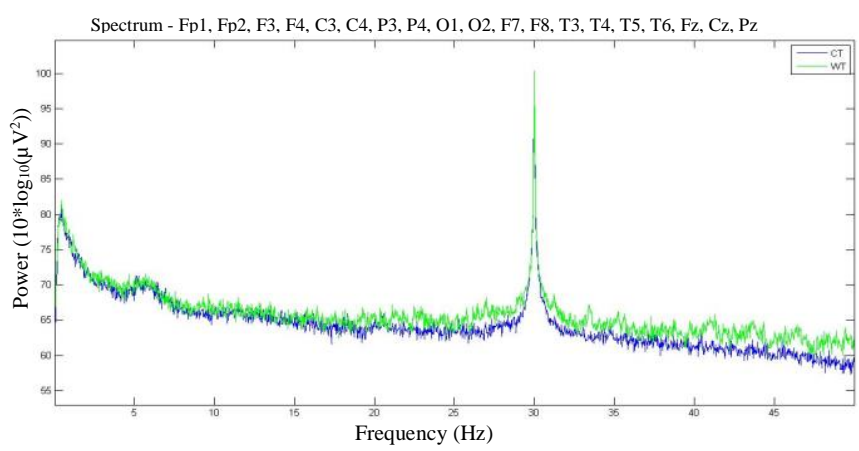

Fig. 5. The brain-wave spectrum in decibel $(\mathrm{dB})$ during colour-task (blue) and word-task (green)

As shown in Fig. 5, in both the colour-task and the wordtask, $30 \mathrm{~Hz}$ frequency signal was strongly recorded compared to other ranges of frequency. The frequency spectrum recorded during word-task was slightly higher than that of the colour-task. Beta-wave frequency also strongly appeared than that of the other types of brain-wave. It means that during both of the tasks, all subjects needed to keep their focus. From 42 EPOCHs which were recorded during the colour- 
task, there were only two weak EPOCHs. Hence, these EPOCHs were removed. This situation occurred because the subject lost his or her concentration. From the same subject, two EPOCHs in the word-task were also removed to make the number of data between the word-task and the colour-task was equal.

Fig. 6 shows the distribution map of $30 \mathrm{~Hz}$ brain-wave frequencies during colour-task (left) and word-task (right) after removing two EPOCHs. It shows that during both of the task, the intensity of $30 \mathrm{~Hz}$ brain-wave around the channels of Fp1 and Fp2 was the highest among the other channels. The other channels that also have high intensity of $30 \mathrm{~Hz}$ brainwave were $\mathrm{P} 4$ and $\mathrm{O} 1$ channels. However, the intensity was lower than that of the Fp1 and Fp2 channels.
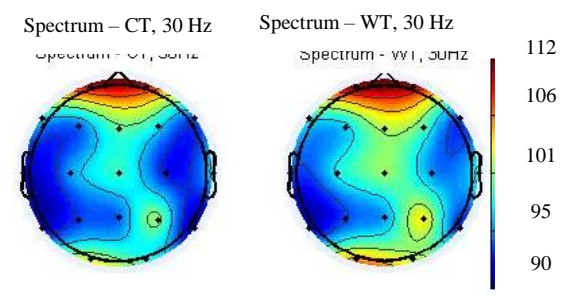

Fig. 6. The distribution of $30 \mathrm{~Hz}$ brain wave frequency that has been recorded during colour-task and word-task

Table 1 shows the $t$-test score between colour-task and word-task in every channel. The $t$-test score threshold was 2.0027 for 40 data with the significance level of $5 \%$. If the $t$ test score of one channel was greater than 2.0027, the activity in that channel during the colour-task was lower than that of the word-task. If the $t$-test score was lower than -2.0027 , the activity in that channel during the colour-task was higher than that of the word-task. If the $t$-test score was between -2.0027 and 2.0027 , the activity in that channel during the word-task was no difference from that of the colour-task.

\subsection{Discussion}

Decision making is more difficult during the colour-task compared to the word-task. It is indicated by the time consumed and the wrong decision produced. It takes from 1.5 to 3 seconds longer to make a decision during the colour-task than that of the word-task. During the word-task, the maximum wrong decision that the subject makes is not more than $2 \%$. While in colour-task, the average of wrong decision is around $21 \%$. This study expects that both of the tasks have the same interference level. However, the results show that the colour-task produced extra interference effect, since the colour-task was more challenging than the word-task.

Both of the tasks show that the beta brain wave was strongly recorded. This study concludes that in the Strooptask, before the decision-making process, the subject needs to concentrate for recognising either the word they read or the colour that they saw. When the subjects conducted the Stroop-task, the subjects needed to concentrate to concentrate on the task for decision-making. Therefore, the beta-wave was strongly active in this study. One of our data did not show the high intensity of beta-wave frequency due to loss of the subject's focus during the undergone task. As a result, the sequence errors have been found in this set of task. Therefore, the decision-making in one case can affect to the next decision-making. The break time between each set was important to reduce the sequential wrong decision-making.

From Table 1, it can be seen that Fp1 and Fp2 channels have higher signal intensity than that of the other channels. Fp1 channel measures the attention focus of the subjects. During the decision making process in both the word-task and the colour-task, the subjects need to preserve their concentration. Therefore, activities of $30 \mathrm{~Hz}$ brain wave in Fp1 channel were strongly recorded because the subjects needed to take all their focus and attention to the task, thus they cannot easily lose their concentration.

Table 1. T-test Score between Colour-task and Word-task for Each Channel

\begin{tabular}{cc}
\hline Channel & T-test Score \\
\hline Fp1 & 3.953 \\
Fp2 & 4.0068 \\
F3 & 2.047 \\
F4 & -0.067 \\
C3 & 2.134 \\
C4 & 0.092 \\
P3 & 1.264 \\
P4 & 2.088 \\
O1 & 1.430 \\
O2 & 1.766 \\
F7 & -0.651 \\
F8 & 0.0977 \\
T3 & -0.473 \\
T4 & 0.921 \\
T5 & -4.15 \\
T6 & 1.851 \\
Fz & 2.601 \\
Cz & 3.448 \\
Pz & 1.061 \\
\hline
\end{tabular}

The activity in Fp2 channel was also strongly recorded in this study because the subjects needed to make a decision in a short time. However, the $t$-test score for Fp1 and Fp2 channels showed that the activity during word-task was higher than that of the colour-task. The colour-task produced higher intensity level than the word-task. Because of the interference level, the subjects needed more time to make a decision during the colour-task than that of the word-task. This study finds that more interference we send to our brain can decrease the accuracy in decision-making results. Accuracy is measured based on the number of correct answers given by the subject according to the given instructions.

Moreover, all of the subjects cannot get a perfect score for colour-task. The result shows that the intensity of the $30 \mathrm{~Hz}$ brain-wave signal during colour-task was lower than wordtask. During this study, the error has affected in subject attention. The number of right and wrong in the monitor tells the subjects whether they make a right decision or wrong decision. All subjects showed that they lost their attention for the task after they knew that they made a wrong decision. Some of the data also showed that this wrong decision had an effect on the next decision-making. Therefore, the intensity of brain-wave in Fp1 channel during colour-task was lower since a lot of errors that they make has affected on their attention 
level. In this study, the wrong decision also affected to another decision-making process and this thing made the 30 $\mathrm{Hz}$ brain-wave during the colour-task lower than the wordtask. This result also relates to ACC working during the decision-making process since the fMRI studies also showed that the interference effect during the incongruent Stroop-task also related to activities in ACC and dorsolateral regions [27].

Although the signal intensities recorded from $\mathrm{P} 4$ and $\mathrm{O} 1$ channels are not as high as that of the Fp1 and Fp2 channels, these channels show that the $30 \mathrm{~Hz}$ frequency has recorded more than that of another channel.

P4 channel is active when someone does the non-verbal reasoning, a human ability to make some responses for some objects that he sees. In this experiment, subjects needed to make a decision based on the case that they see. The visual information makes the brain to do non-verbal reasoning process before they make a decision. The right hemisphere of the brain is the part of the brain that does non-verbal reasoning process. Right hemisphere has the function to understand the information that comes from images or spatial relations. In our study, the subjects needed to make the decision to recognise the colour or the word that they saw, so they needed to extract the visual information before they made the decision. Therefore, the $\mathrm{P} 4$ channel has a record to produce the high signal intensity since the non-verbal reasoning process in this region of the brain.

In subject's brain, the occipital area is the first part of the brain that receives the visual information. In this study, O1 channel has the high result of EEG record because the main role of this part is to extract the information. Before making the decision, the subjects need to choose if they want to recognise the word that they read or that of colour that they see. However, the occipital area recognises both of them, after that the information is continued to ACC and DLPFC region so this part can make the decision. The $t$-test score in O1 channel shows that there is no significant difference in activity at this channel for both of these tasks. It indicates that even the colour-task produces more interference level than that of the word-task, our brain still can do same level of visual information recognition process. In other words, the interference level which was produced in our Stroop-task study did not have any effect for visual recognition process.

In the previous study, there was different activity in motor cortex region during incongruent and congruent Stroop-task [20]. In this study, only left motor cortex region (F3) has different activity during colour-task and word-task ( $t$-test score $=2.1134)$. This study suggests that the incongruent Stroop-task has an effect on both left and right motor cortex. However, since all of the subjects use right-hand to make the response, the left motor cortex has more interference effect than the right motor cortex because the right part of body sensorimotor locates near F3 channel [28].

\section{Conclusion}

In this study, the decision-making is the most important thing to understand. This study concludes that incongruent Stroop-task gives the interference level to disturb the attention and decision-making process. Both of the tasks give high interference level to the subjects. However, this study shows that it is more difficult to recognise the colour than the written word in the Stroop-word. Therefore, the interference level increases when the subjects need to recognise the colour in the Stroop-word. More interference effect produces a harder task, so the time to make a decision during the colour-task is longer than that of the word-task. More interference effect decreases the ability to make a decision. As a result, the more wrong decision appears during the colour-task than that of the wordtask. The wrong decision also disturbs the attention progress of the subjects.

The Stroop-task requires the subjects to concentrate before make a decision. However, the activities of both channels (Fp1 and Fp2) during the colour-task are lower than that of the word-task due to some wrong decisions processes produced in the colour-task.

In this study, the subjects need to extract the visual information. Before the decision-making process, subjects recognise the words that they read and colour that they see. The right-hemisphere in parietal area and the left side of occipital have responsible to do non-verbal reasoning and visual information extraction. However, the amount of interference effect produced in the Stroop-task does not have any effect to information of visual recognition process.

In the next study, the relation between working memory and decision-making process will be investigated. It is also possible to compare the Stroop-task result between normal subjects and Alzheimer subjects. The study has a potential that the Stroop-task can be implemented to slow the decline of brain activity in Alzheimer subjects.

\section{Acknowledgements}

A special thank for Prof. Keiji Iramina as the supervisor of the Neuroimaging and Neuroinformatics Laboratory, Kyushu University Japan. Thank you also for Prof. John Lauweyers for his help to improve our experiment. A beloved thank also for all of the members of Neuroimaging and Neuroinformatics Laboratory. Last but not least, the authors would like to thank the colleagues in Intelligent Systems Research Group in the Department of Electrical Engineering and Information Technology, Faculty of Engineering, Universitas Gadjah Mada.

\section{References}

1. C. M. MacLeod, Half a century of research on the Stroop effect: an integrative review, Psychological bulletin. 109 (1991) 163.

2. J. R. Stroop, Studies of interference in serial verbal reactions, Journal of experimental psychology. 18 (1935) 643.

3. B. S. Peterson, P. Skudlarski, J. C. Gatenby, H. Zhang, A. W. Anderson, and J. C. Gore, An fMRI study of Stroop word-color interference: evidence for cingulate subregions subserving multiple distributed attentional systems, Biological psychiatry. 45 (1999) 1237-1258.

4. S. F. Taylor, S. Kornblum, E. J. Lauber, S. Minoshima, and R. A. Koeppe, Isolation of specific interference processing in the Stroop task: PET activation studies, Neuroimage. 6 (1997) 81-92.

5. C. S. Carter, M. Mintun, and J. D. Cohen, Interference and facilitation effects during selective attention: an H 215 O PET study of Stroop task performance, Neuroimage. 2 (1995) 264-272.

6. J. V. Pardo, P. J. Pardo, K. W. Janer, and M. E. Raichle, The anterior cingulate cortex mediates processing selection in the Stroop attentional 
conflict paradigm, Proceedings of the National Academy of Sciences. 87 (1990) 256-259.

7. M.-A. Vanderhasselt, R. De Raedt, and C. Baeken, Dorsolateral prefrontal cortex and Stroop performance: Tackling the lateralization, Psychonomic bulletin \& review. 16 (2009) 609-612.

8. C. P. Coste, S. Sadaghiani, K. J. Friston, and A. Kleinschmidt, Ongoing brain activity fluctuations directly account for intertrial and indirectly for intersubject variability in Stroop task performance, Cerebral cortex. 21 (2011) 2612-2619.

9. R. Cabeza and L. Nyberg, Imaging Cognition II: An Empirical Review of 275 PET and fMRI Studies, Journal of Cognitive Neuroscience. 12 (2000) $1-47$.

10. D. L. Schomer and F. L. Da Silva, Niedermeyer's electroencephalography: basic principles, clinical applications, and related fields: Lippincott Williams \& Wilkins, 2012.

11. M. D. Rugg and M. G. Coles, Electrophysiology of mind: Event-related brain potentials and cognition: Oxford University Press, 1995.

12. Y. Feng, C.-L. Zhou, J.-C. Zhang, and M.-L. Tian, Neural Mechanisms of Intuitive Tactical Decision-making Predominance of High-level Fencing Athletes, Journal of Medical and Biological Engineering. 30 (2010) $47-$ 56.

13. S. Hoehl and S. Wahl, Recording infant ERP data for cognitive research, Developmental Neuropsychology. 37 (2012) 187-209.

14. G. Schalk, D. J. McFarland, T. Hinterberger, N. Birbaumer, and J. R. Wolpaw, BCI2000: a general-purpose brain-computer interface (BCI) system, IEEE Transactions on biomedical engineering. 51 (2004) 1034 1043.

15. N. Yan, J. Wang, M. Liu, L. Zong, Y. Jiao, J. Yue, et al., Designing a brain-computer interface device for neurofeedback using virtual environments, Journal of Medical and Biological Engineering. 28 (2008) 167-172.

16. C.-T. Lin, F.-C. Lin, S.-A. Chen, S.-W. Lu, T.-C. Chen, and L.-W. Ko, EEG-based brain-computer interface for smart living environmental auto-adjustment, Journal of Medical and Biological Engineering. 30 (2010) 237-245

17. B. Schack, A. C. Chen, S. Mescha, and H. Witte, Instantaneous EEG coherence analysis during the Stroop task, Clinical Neurophysiology. 110 (1999) 1410-1426
18. D. Szücs, C. Killikelly, and S. Cutini, Event-related near-infrared spectroscopy detects conflict in the motor cortex in a Stroop task, Brain research. 1477 (2012) 27-36.

19. C. Nombela, M. Nombela, P. Castell, T. García, J. López-Coronado, and M. T. Herrero, Alpha-Theta Effects Associated with Ageing during the Stroop Test, PloS one. 9 (2014) e95657.

20. R. Cabeza, S. M. Daselaar, F. Dolcos, S. E. Prince, M. Budde, and L. Nyberg, Task-independent and task-specific age effects on brain activity during working memory, visual attention and episodic retrieval, Cerebral cortex. 14 (2004) 364-375

21. J. M. Bugg, E. L. DeLosh, D. B. Davalos, and H. P. Davis, Age differences in Stroop interference: Contributions of general slowing and task-specific deficits, Aging, Neuropsychology, and Cognition. 14 (2007) 155-167.

22. C. Michel, D. Lehmann, B. Henggeler, and D. Brandeis, Localization of the sources of EEG delta, theta, alpha and beta frequency bands using the FFT dipole approximation, Electroencephalography and clinical neurophysiology. 82 (1992) 38-44

23. W. Herrmann, K. Fichte, and G. Freund, Reflections on the topics: EEG frequency bands and regulation of vigilance, Pharmacopsychiatry. 12 (1979) 237-245.

24. A. A. Fingelkurts and A. A. Fingelkurts, EEG oscillatory states: universality, uniqueness and specificity across healthy-normal, altered and pathological brain conditions, PLoS One. 9 (2014) e87507.

25. S. Zysset, K. Müller, G. Lohmann, and D. Y. von Cramon, Color-word matching Stroop task: separating interference and response conflict, Neuroimage. 13 (2001) 29-36.

26. A. Delorme and S. Makeig, EEGLAB: an open source toolbox for analysis of single-trial EEG dynamics including independent component analysis, Journal of neuroscience methods. 134 (2004) 9-21.

27. D. Floden, A. Vallesi, and D. T. Stuss, Task context and frontal lobe activation in the Stroop task, Journal of Cognitive Neuroscience. 23 (2011) 867-879.

28. J. Duff, The usefulness of quantitative EEG (QEEG) and neurotherapy in the assessment and treatment of post-concussion syndrome, Clinical EEG and Neuroscience. 35 (2004) 198-209. 